\title{
Improving your experience with electronic health records
}

\author{
Steven Lippmann, MD
}

Dr. Lippmann is Emeritus Professor, Department of Psychiatry, University of Louisville School of Medicine, Louisville, Kentucky.

\section{Disclosure}

The author reports no financial relationships with any companies whose products are mentioned in this article, or with manufacturers of competing products.

doi: 10.12788/cp.0030

\section{$f$}

Discuss this article at www.facebook.com/ MDedgePsychiatry 6
$\mathrm{T}$ he electronic health record (EHR) was introduced to improve how clinicians document patient information, contribute to medical research, and allow for medical records to be universally transferable. ${ }^{1}$ However, many clinicians find EHRs to be burdensome, time-consuming, and inefficient. Clinicians often spend multiple hours each day navigating their EHR system, which reduces the amount of time they spend interacting with patients and contributes to physician burnout. ${ }^{1-3}$ For example, in a study of 142 family medicine physicians, clinicians reported that they spent approximately 6 hours per work day interacting with their EHR. ${ }^{3}$

Clearly, the EHR needs a fundamental revision. In the meantime, how can we adapt to improve the situation? Here I suggest practical steps clinicians can take to improve their experience with their EHR system. ${ }^{4-8}$

\section{Steps to take during patient visits}

Because entering information into the EHR can be distracting, be prepared to multitask during each clinical encounter. ${ }^{1-7}$ Be ready to address pertinent inquiries and issues your patient raises, and provide instructions on therapies and interventions. Because interpersonal relations are important during clinical encounters, establish interaction with your patient by acknowledging them and maintaining frequent eye contact.' Consider allowing your patient to view the EHR screen because doing so might increase his/her involvement in the visit.

So that you can pay closer attention to your patient, consider taking notes during the visit and entering the information into the EHR later. Consider improving your typing skills to increase the speed of your note-taking. Alternatively, using a voicerecognition recording tool to transcribe your notes via speech might help you spend less time on note-taking. ${ }^{3}$ Whenever possible, finish charting for one patient before meeting with the next because doing so will save time and help you to better remember details. ${ }^{7}$

In addition, lowering your overall stress might help reduce the burden of using the EHR. ${ }^{3-5}$ Adopt healthy behaviors, including good sleep, nutrition, exercise, and hobbies, and strive for balance in your routines. Attend to any personal medical or psychiatric conditions, and avoid misusing alcohol, medications, or other substances.

\section{Optimize how your practice functions}

With your clinical group and colleagues, create a comfortable environment, good patient-to-doctor interactions, and a smooth flow within the practice. Simplify registration. Ask patients to complete screening forms before an appointment; this information could be entered directly into their EHR. ${ }^{3}$ Consider using physician-extender staff and other personnel, such as scribes, to complete documentation into the EHR. ${ }^{3,8}$ This may help reduce burnout, create more time for clinical care, and improve faceto-face patient interactions. ${ }^{8}$ Employing scribes can allow doctors to be better able to directly attend to their patients while complying with record-keeping needs. Although scribes make charting easier, they are an additional expense, and must be trained. 


\section{Consider EHR training}

EHR training sessions can teach you how to use your EHR system more efficiently. ${ }^{6}$ Such education may help boost confidence, aid documentation, and reduce the amount of time spent correcting coding errors. In a study of 3,500 physicians who underwent a 3-day intensive EHR training course, $85 \%$ to $98 \%$ reported having improved the quality, readability, and clinical accuracy of their documentation. ${ }^{6}$

\section{Help shape future EHRs}

Individual doctors and professional groups can promote EHR improvements through their state, regional, and / or national organizations and medical societies. These bodies should deliver EHR revision recommendations to government officials, who can craft laws and regulations, and can influence regulators and/or insurance companies. Clinicians also can communicate with EHR developers on ways to simplify the usability of these tools, such as reducing the amount of steps the EHR's interface requires. ${ }^{5}$ With a more efficient EHR, we can better concentrate on patient care, which will reduce expenses and should yield better outcomes.

\section{References}

1. Ehrenfeld JM, Wonderer JP. Technology as friend or foe? Do electronic health records increase burnout? Curr Opin Anesthesiol. 2018;31(3):357-360.

2. Meigs SL, Solomon M. Electronic health record use a bitter pill for many physicians. Perspect Health Inf Manag. 2016;13:1d.

3. Arndt BG, Beasley JW, Watkinson MD, et al. Tethered to the EHR: primary care physician workload assessment using EHR event log data and time-motion observations. Ann Fam Med. 2017;15(5):419-426.

4. Fogarty CT, Winters P, Farah S. Improving patient-centered communication while using an electronic health record: report from a curriculum evaluation. Int J Psych Med. 2016;51(4):379-389.

5. Guo U, Chen L, Mehta PH. Electronic health record innovations: helping physicians - one less click at a time. Health Inf Manag. 2017;46(3):140-144

6. Robinson KE, Kersey JA. Novel electronic health record (EHR) education intervention in large healthcare organization improves quality, efficiency, time, and impact on burnout. Medicine. 2018;91(38):e123419. doi: 10.1097/ MD.0000000000012319.

7. Fogarty CT. Getting your notes done on time. Fam Pract Manag. 2016;23(2):40.

8. DeChant PF, Acs A, Rhee KB, et al. Effect of organizationdirected workplace interventions on physician burnout: a systematic review. Mayo Clin Proc Innov Qual Outcomes. 2019;3(4):384-408.
Consider allowing patients to view the EHR screen because doing so might increase their involvement in the visit

\section{Pry open an issue of CURRENT PsYCHIATRY... ... and you might find your Pearl}

\section{Think back on the lessons of years in practice, and share your wisdom in a Current Psychiatry Pearl.}

CURRENT Psychiatry Pearls are practical, focused, and always popular articles in which readers tell their peers how they've learned to tackle everyday clinical and practice management challenges: calm an angry patient, prevent theft of prescription pads, deal with the aftermath of a patient's suicide, readily obtain informed consent, and so on.

Begin by reading the Submission Guidelines at MDedge.com/psychiatry/page/pearls.

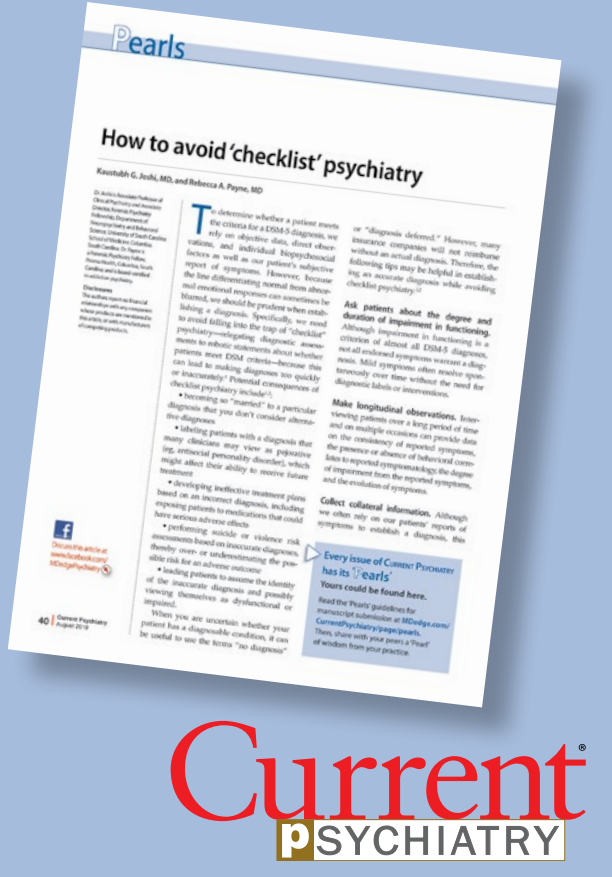

